\title{
Inhibiting $\boldsymbol{\beta}$-catenin expression promotes efficiency of radioiodine treatment in aggressive follicular thyroid cancer cells probably through mediating NIS localization
}

\author{
LING LAN ${ }^{1}$, SPYROS BASOURAKOS $^{2}$, DAI CUI $^{3}$, XUEMEI ZUO $^{2}$, WEI DENG $^{1}$, LILI HUO $^{1}$, \\ LINGHAI CHEN $^{1}$, GUOYING ZHANG ${ }^{1}$, LILI DENG ${ }^{1}$, BINGYIN SHI ${ }^{4}$ and YONG LUO ${ }^{2,5}$ \\ ${ }^{1}$ Department of Endocrinology, Beijing Jishuitan Hospital, Beijing 100035, P.R. China; ${ }^{2}$ Department of Genitourinary, \\ Cancer Medicine, MD Anderson Cancer Center, The University of Texas, Houston, TX 77030, USA; \\ ${ }^{3}$ Department of Endocrinology, First Affiliated Hospital of Nanjing Medical University, Nanjing, Jiangsu 210029; \\ ${ }^{4}$ Department of Endocrinology, The First Affiliated Hospital of Medical College of Xi'an Jiaotong University, Xi'an, \\ Shaanxi 710061; ${ }^{5}$ Department of Urology, Beijing Anzhen hospital, Capital Medical, University, Beijing 100029, P.R. China
}

Received May 26, 2016; Accepted October 18, 2016

DOI: $10.3892 /$ or.2016.5228

\begin{abstract}
The present study investigated whether the efficacy of radioiodine therapy towards aggressive thyroid cancer cells was affected by $\beta$-catenin activity and associated with sodium/iodine symporter (NIS) localization. Human thyroid cancer cell line follicular thyroid carcinoma (FTC) 133 was endowed with aggressiveness by HIF-1 $\alpha$ or $\beta$-catenin overexpression. The protein amount and subcellular localization of NIS, and the radioiodine uptake capacity were detected in the cells, as well as in cells subsequently undergoing $\beta$-catenin knockdown. Xenograft experiments were conducted to compare the tumor growth ability and responsiveness to radioactive treatment among HIF- $1 \alpha$ and $\beta$-catenin overexpressing FTC cells, respectively with or without $\beta$-catenin knockdown. $\beta$-catenin increased upon HIF-1 $\alpha$ overexpression, but not vice versa. This signal axis would prompt metastatic propensity in FTC cells, and translocate NIS from cytomembrane to cytoplasm. Consistently the radioiodine uptake capacity in the cells decreased obviously. Knockdown of $\beta$-catenin reversed all these changes. Furthermore, the xenograft experiments showed that radioiodine treatment could thoroughly suppress tumor growth ability of aggressive FTC cells only
\end{abstract}

Correspondence to: Dr Ling Lan, Department of Endocrinology, Beijing Jishuitan Hospital, Beijing 100035, P.R. China

E-mail: lanling2016@sina.com

Dr Yong Luo, Department of Genitourinary, Cancer Medicine, MD Anderson Cancer Center, The University of Texas, Houston, TX, 77030, USA

E-mail: luoyongdoctor@163.com

Key words: thyroid cancer, sodium-iodine symporter, radioiodinerefractory, $\beta$-catenin, HIF-1 $\alpha$ if the HIF- $1 \alpha$-induced $\beta$-catenin activation was disrupted by $\beta$-catenin knockdown. $\beta$-catenin nuclear translocation in tumor cells was accompanied by abnormal subcellular localization of NIS. Moreover, we found that only after inhibiting $\beta$-catenin expression, can the radioiodine treatment promote apoptosis other than repress proliferation and survival in xenograft tumor cells. In conclusion, aggressive FTC cells overexpressing HIF-1 $\alpha$ will be fully cracked down by radioiodine therapy once $\beta$-catenin expression is inhibited, and regulated localization of NIS may account for underlying mechanisms.

\section{Introduction}

Thyroid cancer is one of the most common types of endocrine malignancy, with a gradual increase in the incidence rate; and it is the fifth leading cause of cancer globally, accounting for $2.1 \%$ of all the newly detected cancers $(1,2)$. Most of the cases of thyroid cancers show biologically indolent phenotype and have an excellent prognosis with survival rates of more than $95 \%$ at 20 years although the recurrence or persistent rate is still high (2).

As classified by World Health Organization (WHO), most primary thyroid cancers are epithelial tumors that originate from thyroid follicular cells, comprising three main pathological types: papillary thyroid carcinoma (PTC), follicular thyroid carcinoma (FTC) and anaplastic thyroid carcinoma (ATC). While another kind of epithelial tumor, medullary thyroid carcinoma (MTC) arises from thyroid parafollicular cells. PTC and FTC are categorized as differentiated thyroid cancer (DTC), they are well-differentiated and have indolent tumor growth (3). They account for $>90 \%$ of thyroid cancers, while MTC only accounts for $<5 \%$ (4).

Radioiodine is a safe and effective modality used for more than 60 years in treating thyroid cancers and has potential uses in breast, prostate and other cancers. ${ }^{131} \mathrm{I}$ is generally used in radiotherapy and for in vivo experiments because its half-life 
of merely 8 days (5). Ablative radioiodine therapy exploits the ability of the thyroid to take up iodide, a process mediated by the sodium iodide symporter (NIS), which is an integral membrane glycoprotein located in the basolateral plasma membrane of thyroid follicular epithelial cells $(6,7)$. However, thyroid tumors such as FTC have a chance of developing aggressive thyroid carcinomas with metastatic propensity and resistance to radioiodine (5,8-11). As it is more likely to produce distant metastases, FTC may have a poorer prognosis compared with PTC (10-12).

It is necessary to identify the underlying mechanisms leading to decreased efficiency of radioiodine therapy in those radioiodine-refractory thyroid tumors. As mentioned above, for active iodide uptake to occur, adequate amount of NIS must be located in the cytomembrane of thyroid follicular epithelial cells (13). Numerous researchers have focused on finding ways to increase NIS expression in thyroid cancers, with expectation of improving the iodide transport ability in tumor cells and elevating the effectiveness of radioiodine therapy $(14,15)$. However, according to some other studies, NIS is overexpressed in some cases of thyroid cancers (detected by immunohistochemistry), wherein the impaired function of NIS may be attributed to its abnormal internalization $(7,13-$ 20). Thus, it may be the post-translational regulation rather than the decreased expression of NIS that robustly contribute to a reduction in iodide uptake in some thyroid cancers $(21,22)$. Therefore, it is of great importance to explore the factors taking part in regulating the NIS subcellular localization, especially in the cases of aggressive thyroid carcinomas resistance to radioiodine therapy.

Catenin beta-1 ( $\beta$-catenin) is a dual function protein regulating cell-cell adhesion as well as transcription of number of genes, the latter of which is mainly controlled by Wnt signaling molecules (canonical Wnt pathway) (23). In 2014, SastrePerona and Santisteban (24) revealed the Wnt-independent role of $\beta$-catenin in regulating the proliferation and differentiation of rat thyroid follicular cells. They found that direct interaction of $\beta$-catenin with Pax 8 resulted in increased activity of NIS. According to their conclusion, $\beta$-catenin is possibly a positive regulator of NIS and iodide uptake activity in physiological conditions. On the other hand, studies have shown that $\beta$-catenin was overexpressed in aggressive thyroid cancers (25-28). Whether $\beta$-catenin plays a critical role in affecting the expression of NIS within thyroid tumor cells, as well as regulating the abnormal subcellular localization, is worthy of investigation.

Previous studies have discovered that $\beta$-catenin can enhance HIF-1 mediated transcription events, thus, promoting cancer cell survival and endowing them with better adaptability to hypoxia $(23,25,29,30)$. HIF are associated with poor prognosis in many tumor types $(31,32)$. Like $\beta$-catenin, the overexpression of HIF-1 $\alpha$ was also observed in thyroid cancers, and the potential significance of the HIF signaling pathway in progression and aggressiveness of thyroid carcinoma has been demonstrated (33). Compared with that in normal thyroid tissues, HIF-1 $\alpha$ expression was significantly greater in various types of primary thyroid tumors and ATC and FTC showed the highest levels of expression (34). Moreover, HIF-1 $\alpha$ may reduce the effectiveness of cancer treatment as it has been shown that overexpression of HIF-1 $\alpha$ in cancer cells plays an important role in the development of resistance to radiotherapy and some forms of chemotherapy (35).

We presume that HIF-1 $\alpha$ may exert its power on desensitisation to radiotherapy in aggressive thyroid carcinomas in $\beta$-catenin-dependent manner. This signal pathway may act through changing membrane localization other than transcriptional level of NIS. Therefore, in the present study, we examined the subcellular localization of NIS in relation to $\beta$-catenin nuclear translocation, subsequent to the activation of HIF signaling pathway in FTC cells. We also disrupted the $\beta$-catenin signal in the regulatory mechanism to see if the efficacy of radioiodine treatment in HIF-1 $\alpha$ overexpressing aggressive thyroid cancers is promoted. Our data suggest an entirely novel mechanism that alters NIS subcellular localization, thereby regulating cell ability to aggressively uptake iodide in cancer cells.

\section{Materials and methods}

Cell culture. Human thyroid carcinoma cell line FTC133, which was derived from the primary tumor of a 42-year-old male patient with metastatic FTC (36) was kindly provided by Professor Karl-M. Derwahl (Humboldt University, Berlin, Germany). The FTC133 cells were cultured in Dulbecco's modified Eagle's medium (DMEM; Invitrogen, Carlsbad, CA, USA) supplemented with $10 \%$ fetal bovine serum (FBS; Sigma-Aldrich, St. Louis, MO, USA) and penicillin/ streptomycin in a humidified incubator (Thermo Scientific Forma 370; Thermo Fisher Scientific, Shanghai, China) at $37^{\circ} \mathrm{C}$ and $5 \% \mathrm{CO}_{2}$.

Plasmids transfection. Recombinant plasmid pcDNA3.1(-)/ HIF-1 $\alpha$ was kindly provided by Professor Leland W.K. Chung from the Winship Cancer Center at Emory University (Atlanta, GA, USA). Recombinant plasmids pcDNA3.1()$/ \beta$-catenin and pSUPER/ $\beta$-catenin were constructed by $\mathrm{Dr}$ Yong Luo in our laboratory, the latter of which was used for shRNA mediated knockdown of $\beta$-catenin as previously described (37).

FTC133 cells were transfected with pcDNA3.1 (-)/HIF-1 $\alpha$, pcDNA3.1 (-)/ $\beta$-catenin by means of Lipofectamine 2000 system (Invitrogen, Carlsbad, CA, USA). After a 6 h co-incubation, the cells were then cultured for 2 days with DMEM (containing 10\% FBS); after that, the cells were passaged (1:3) and then screened with the selective medium (containing $400 \mu \mathrm{g} / \mathrm{ml} \mathrm{G} 418$ ); selective medium was replaced every 5 days. Four weeks later, all the untransfected cells died by apoptosis and transfected cells formed into monoclonal cell clusters; the cells were maintained in the medium containing $200 \mu \mathrm{g} / \mathrm{ml}$ G418. The acquired stably transfected lines were named as FTC133-HIF-1 $\alpha$ and FTC133- $\beta$-catenin, respectively.

For shRNA-mediated knockdown, FTC133-HIF-1 $\alpha$ and FTC133- $\beta$-catenin cells were transfected with pSUPER/ $\beta$-catenin by means of Lipofectamine 2000 (Invitrogen). After a $6 \mathrm{~h}$ co-incubation, the cells were then cultured for 3 days with DMEM (containing 10\% FBS); then the cells were passaged $(1: 3)$ and screened with the selective medium (containing $400 \mu \mathrm{g} / \mathrm{ml}$ puromycin) for 4 weeks. All the untransfected cells died by apoptosis and transfected cells formed into monoclonal 
cell clusters; the silent cells were maintained in the medium containing $200 \mu \mathrm{g} / \mathrm{ml}$ puromycin.

Western blotting. Cell lysate preparation and blotting conditions have been previously described (4). Antibodies (Gibco, Waltham, MA, USA) against HIF- $1 \alpha, \beta$-catenin, NIS and GAPDH (as an internal control) were used for western blot analyses as per instructions of the manufacturers.

Immunofluorescent staining. Cells were fixed in $10 \%$ paraformaldehyde for $30 \mathrm{~min}$ and blocked with goat serum (Gibco) for $30 \mathrm{~min}$, respectively. Cells were then incubated at $37^{\circ} \mathrm{C}$ for $1 \mathrm{~h}$ with the rabbit anti-human $\beta$-catenin primary antibody or the rabbit anti-human NIS antibody (Santa Cruz Biotechnology, Santa Cruz, CA, USA), at a dilution of $1: 100$ in $0.5 \%$ BSA in phosphate-buffered saline (PBS). After washing 3 times with PBS, cells were co-incubated with the appropriate rhodamine or fluorescein-tagged goat anti-rabbit antibody (Sigma-Aldrich), diluted at $1: 50$ in $0.5 \%$ BSA in PBS at $37^{\circ} \mathrm{C}$ for $1 \mathrm{~h}$. After final washes with PBS, the coverslips were mounted on a microscope slide. The fluorescence staining intensity and intracellular localization were then examined by Olympus immunofluorescence microscope (BX43).

Iodide uptake assay. ${ }^{125} \mathrm{I}$ is generally applied during iodide uptake assay since its half-life is as long as 60 days $(5,10)$.

Cells were grown as monolayer on 6 -well plates at a concentration of $5 \times 10^{4}$ cells/well. To measure the iodide uptake activity, after 3-4 days of culture, cells were washed with HBSS and incubated in the reaction buffer containing ${ }^{125} \mathrm{I}$ (3.7 $\mathrm{KBq}$ ) (Yuanzi, China) for $20 \mathrm{~min}$ at $37^{\circ} \mathrm{C}$, and cells incubated with the solvent (water-free alcohol) alone was used as control. After incubation, cells were washed with ice-cold HBSS 3 times, and then incubated with $1 \mathrm{ml}$ ice-cold water-free alcohol for $20 \mathrm{~min}$. The radioactivity was measured for the incubation water-free alcohol in a $\gamma$-counter (Wizard 2480; Perkin-Elmer, Waltham, MA, USA), as previously described (38). ${ }^{125}$ I-uptake was expressed as counts per minute.

MTT assay. Cells $\left(1 \times 10^{4}\right)$ were seeded into each well of 96-well tissue culture plates. At various time-points (24, 48 and $72 \mathrm{~h})$ of cell culture in DMEM, $50 \mu 1$ MTT solution $(2.5 \mathrm{mg} / \mathrm{ml})$ was added into the plate and incubated with cells for an additional $4 \mathrm{~h}$. The media were collected separately from each chamber, and cell-associated MTT crystals were dissolved separately in dimethyl sulfuroxide (DMSO; $150 \mu \mathrm{l} /$ well) on a shaker at room temperature. The color intensity was measured at $570 \mathrm{~nm}$ against the appropriate blank controls ( $0.1 \%$ BSA/RPMI1640 medium with MTT solution and $150 \mu \mathrm{l}$ DMSO) using a Bio-Rad Technologies microplate reader.

Invasion assay. Cells were cultured in 6-well plates to $90 \%$ confluency and then collected after trypsinization. An analysis assessing the invasion of cells was performed using 6-well Transwell inserts with $6.5-\mathrm{mm}$ diameters and $8-\mu \mathrm{M}$ pores (Corning, Corning, NY, USA). In brief, the filters were precoated for $30 \mathrm{~min}$ at $37^{\circ} \mathrm{C}$ with $25 \mu 1$ extracellular matrix (Sigma-Aldrich) gel mixed with dimethyl sulfoxide (1:1). The trypsinised cells $\left(7 \times 10^{4}\right)$ were washed with PBS, resuspended in the serum-free medium, and placed in the upper chamber, and a medium containing $10 \%$ FBS was used as a chemoattractant in the lower chamber. Cells were incubated at $37^{\circ} \mathrm{C}$ in $5 \% \mathrm{CO}_{2}$ for 24,48 and $72 \mathrm{~h}$, respectively, and the number of cells that invaded across the membranes were fixed and stained with Giemsa. The non-migratory cells on the upper chamber were removed with cotton swabs, and the migratory cells present on the lower surface were counted in 10 random fields and photographed at x100 magnification under an inverted microscope (Olympus).

Xenograft experiments. This part of the study was carried out in strict accordance with the recommendations in the Guide for the Care and Use of Laboratory Animals in the Weatherall Report. In addition, the protocol was approved by the Ethics Committee for Animal Experiments of the Beijing University. Surgery was performed under sodium pentobarbital (Merk, Darmstadt, Germany) anesthesia, and all efforts were made to ameliorate suffering.

Six-week-old male SCID mice (Charles River Laboratories, Wilmington, MA, USA) were injected subcutaneously with $2 \times 10^{6}$ cells in the left forelimb. Animals (8-10 per group) were monitored daily, and tumor volumes were measured by vernier caliper with the formula: $\mathrm{V}=\left(\mathrm{LW}^{2}\right) / 2$, where $\mathrm{L}$ is the length and $\mathrm{W}$ is the width. When xenograft tumor grew to the volume of $50 \mathrm{~mm}^{3}$, mice of the experiment groups (see the corresponding figure legends for the specific group settings) were treated with peritoneal injection of $37 \mathrm{MBq}{ }^{131} \mathrm{I}$, whereas those of control groups were injected with $0.1 \mathrm{ml}$ physical saline. Thirty-five days after the radioiodine treatment, tumors were weighed and fixed overnight at $4{ }^{\circ} \mathrm{C}$ in $10 \%$ paraformaldehyde and embedded in paraffin for histological analysis.

Immunohistochemical staining. Paraffin-embedded sections of xenograft cancer tissues were used for immunohistochemical staining, using a commercially available kit (Boshide, Wuhan, China). Primary antibodies (Sigma-Aldrich) against $\beta$-catenin, NIS, cell cycle protein (Ki-67), anti-apoptosis protein (survivin) and apoptosis protein (caspase-3) were used. The samples were then counter stained with hematoxylin to indicate the nucleus.

The results were observed by three physicians, who were blinded to the grouping. For every index, 3 pictures of cancer tissues from each animal $(n=10$, per group) were selected for quantification. Five high power fields were selected from each picture to assess the percentage of positively staining cells in all fields of observation.

Statistical analysis. Statistical analyses were performed using the SPSS statistical software 13.0. The data are represented as mean \pm standard derivation (SD). Statistical significance was evaluated by one-way analysis of variance (ANOVA) with the Student-Newman-Keuls (SNK) test for post hoc test or Student's t-test. $\mathrm{P}<0.05$ was considered statistically significant.

\section{Results}

HIF-1 $\alpha$ overexpression endowed FTC cells with aggressiveness in a $\beta$-catenin-dependent manner. As shown clearly by western blotting (Fig. 1A), HIF-1 $\alpha$ overexpression upregulated $\beta$-catenin in FTC cells, but not vice versa. Application of 

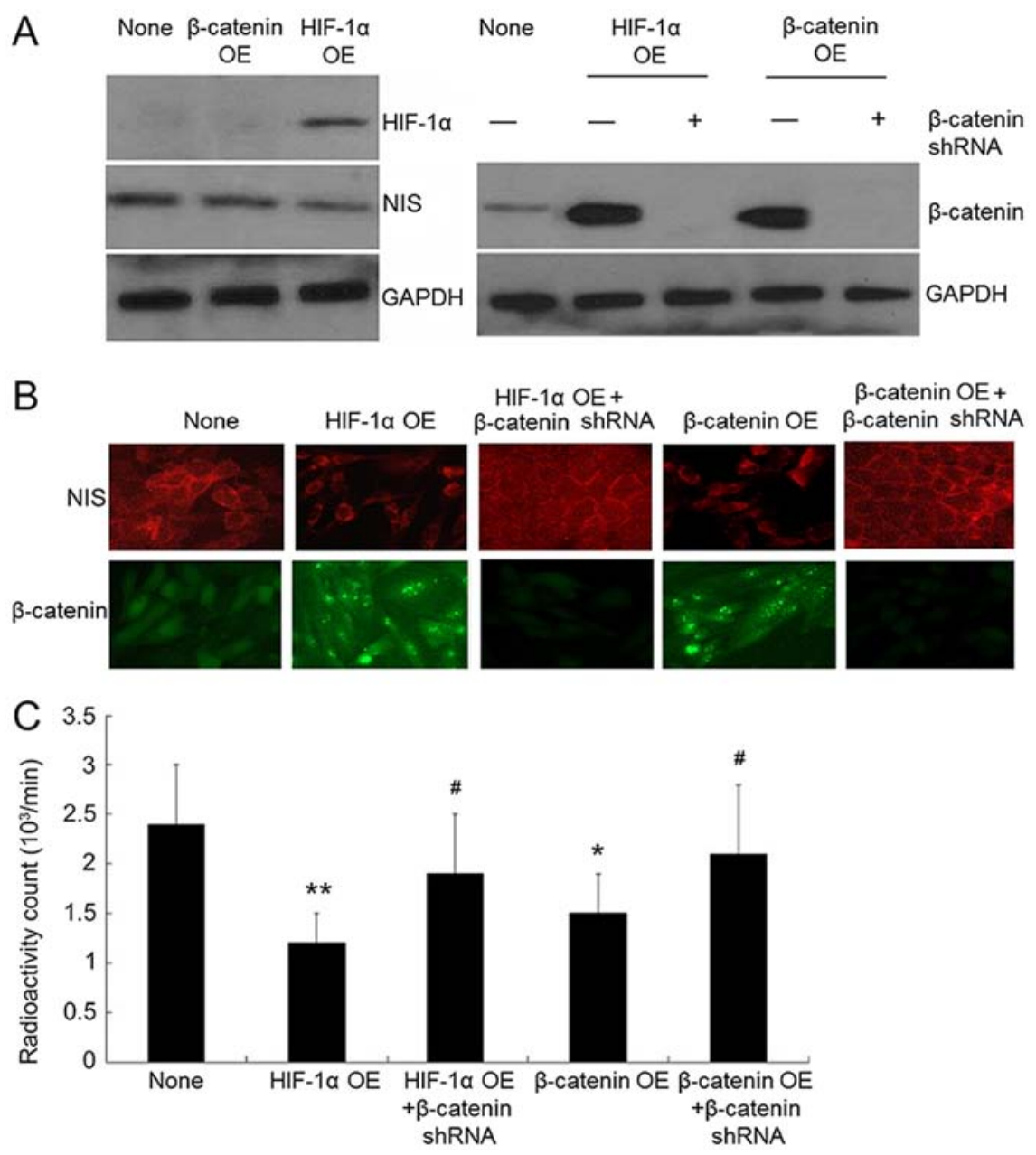

Figure 1. $\beta$-catenin activation subsequent to HIF-1 $\alpha$ overexpression changes NIS subcellular localization and impair cell radioiodine uptake. The overexpression (OE) of HIF-1 $\alpha$ or $\beta$-catenin in FTC133 cells was by pcDNA3.1, and the shRNA of $\beta$-atenin was by pSUPER. FTC133 cells without plasmid transfection were defined as the None group and used as control. (A) Representative western blotting of HIF-1 $\alpha$, $\beta$-catenin, or NIS from FTC133 cells transfected with different plasmids or none. GAPDH was used as the internal control. (B) Representative immunofluorescent staining of $\beta$-catenin or NIS in FTC133 cells transfected with different plasmids or none. Magnification, $\mathrm{x} 400$. (C) The radioiodine uptake ability of FTC133 cells transfected with different plasmids or none. The solvent in the radioiodine uptake assay was alcohol. Each assay was performed as one experiment in triplicate for each group, except the radioiodine uptake assay where two independent experiments (each one in triplicate) were carried out. ${ }^{*} \mathrm{P}<0.05$ and ${ }^{* *} \mathrm{P}<0.01$ vs. the None group. ${ }^{*} \mathrm{P}<0.05$ between the same OE groups with and without $\beta$-catenin shRNA knockdown.

$\beta$-catenin shRNA can successfully reduce the protein amount of $\beta$-catenin.

FTC cells overexpressing either HIF- $1 \alpha$ or $\beta$-catenin were compared for changes in the content of several markers concerned with epithelial-mesenchymal transitions (EMT) (Fig. 2A). There is a decrease in the levels of epithelial markers E-cadherin and CK18 but an increase in the levels of vimentin, VEGF, fibronectin and MMP-2 in the FTC cells overexpressing either HIF- $1 \alpha$ or $\beta$-catenin. However, these EMT phenotypes were all restored after knockdown of $\beta$-catenin.

We further found that HIF-1 $\alpha / \beta$-catenin overexpression will favor FTC cells with stronger growth ability as well as metastatic propensity (Fig. 2B and C). Whereas, once the $\beta$-catenin expression was reduced by shRNA knockdown, the changes in the aggressiveness of the thyroid cancer cells were reversed.

$\beta$-catenin activation subsequent to HIF-1 $\alpha$ overexpression changes NIS subcellular localization and impair cell radioiodine uptake. As evident in western blotting (Fig. 1A), HIF-1 $\alpha$ or $\beta$-catenin overexpression results in a mild decrease in the content of NIS. From immunofluorescent staining (Fig. 1B), we found that additional to the protein amount, HIF-1 $\alpha$ overexpression also promoted the nuclear translocation of $\beta$-catenin, and relocated NIS from the originally diffuse distribution or location near the cytomembrane into intracellular localization near the nucleus. These changes of protein localization also appear when $\beta$-catenin was directly overexpressed, and were reversed by $\beta$-catenin knockdown.

Additionally, cell radioiodine uptake assay (Fig. 1C) showed that overexpression of either HIF-1 $\alpha$ or $\beta$-catenin decreased radioiodine uptake ability of FTC cells $\sim 50$ and $37.5 \%$, respectively $(\mathrm{P}<0.05$ or $\mathrm{P}<0.01$, respectively). While after $\beta$-catenin knockdown, the impaired iodide uptake capacity was restored (all $\mathrm{P}<0.05$ ).

$\beta$-catenin knockdown can improve the growth inhibitory effect of radioiodine treatment in HIF-1 $\alpha$ overexpressing FTC cells. In the xenograft tumor model, we found that overexpression of HIF-1 $\alpha$ and $\beta$-catenin could both promote the growth 
A
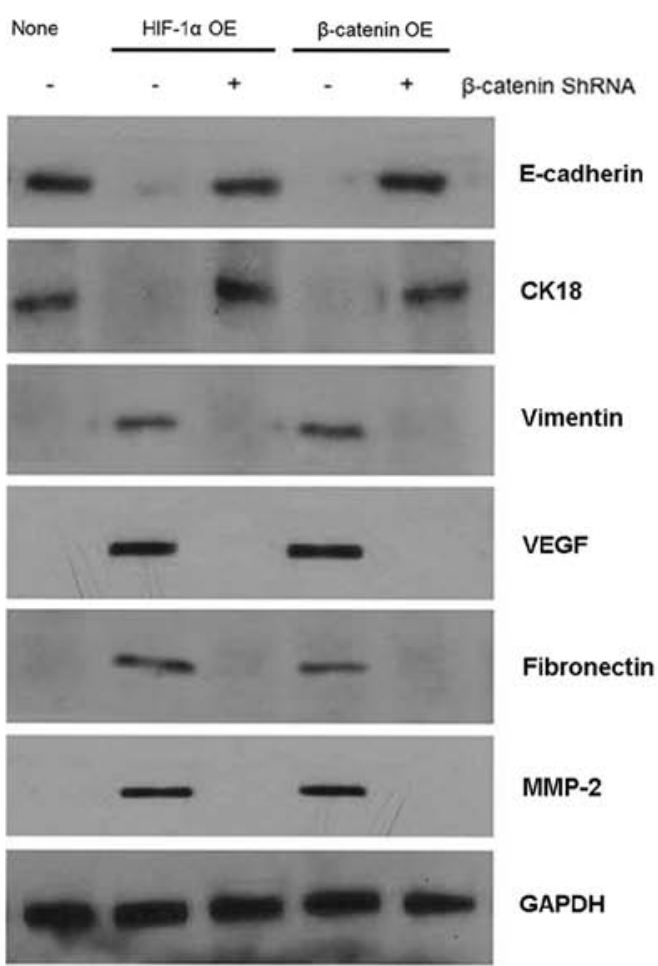

Vimentin

VEGF

Fibronectin

MMP-2

GAPDH
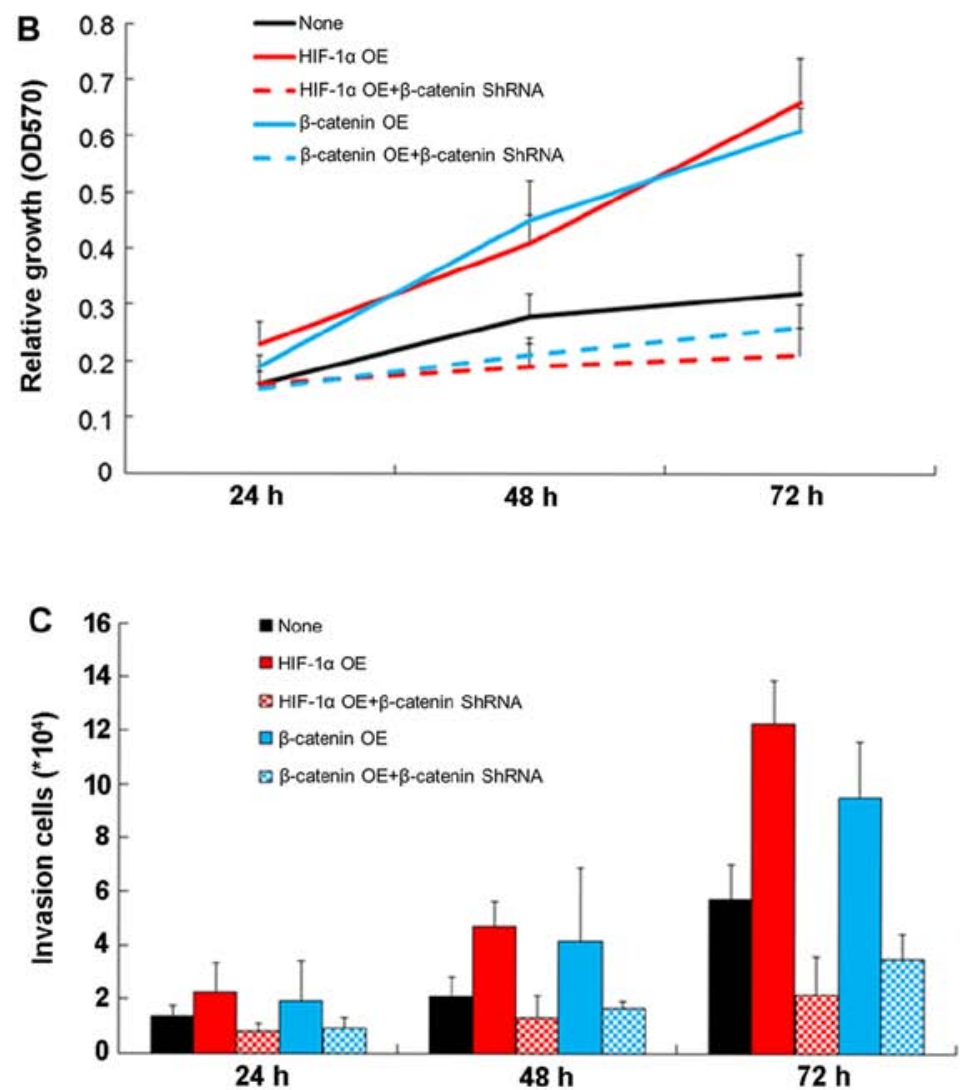

Figure 2. HIF-1 $\alpha$ overexpression endowed FTC cells with aggressiveness in a $\beta$-catenin-dependent manner. The overexpression (OE) of HIF-1 $\alpha$ or $\beta$-catenin in FTC133 cells was by pcDNA3.1, and the shRNA of $\beta$-catenin was by pSUPER. FTC133 cells without plasmid transfection were defined as the None group and used as control. (A) Representative western blotting of epithelial-mesenchymal transitions (EMT) markers from FTC133 cells transfected with different plasmids or none. GAPDH was used as the internal control. (B) The growth viability of FTC133 cells transfected with different plasmids or none were measured by MTT assay. The detection time-points were 24, 48 and 72 h during cell culture. (C) The metastatic potential of FTC133 cells transfected with different plasmids or none were measured by invasion assay. The detection time-points were 24,48 and 72 h during cell culture. Each experiment was made in triplicate for each group.

of FTC cells (Fig. 3A), while ${ }^{131}$ I treatment inhibited such tumor growth. Quantification of tumor mass at 35 days after radiotherapy (right in Fig. 3A) also showed that ${ }^{131} \mathrm{I}$ treatment could markedly kill the HIF-1 $\alpha$ overexpressing cancer cells or inhibit their growth, but further combined with $\beta$-catenin knockdown significantly promoted the therapeutic efficacy $(\mathrm{P}<0.05)$.

Importantly, immunohistochemical analysis demonstrated that (Figs. 3C and 4), overexpression of HIF-1 $\alpha$ will significantly promote the expression of Ki-67 and survivin in the thyroid cancer cells as well as inhibit the expression of caspase-3. After ${ }^{131} \mathrm{I}$ treatment in the HIF-1 $\alpha$ overexpressing FTC cells, Ki-67 was significantly reduced, and survivin had a moderate decline, but the level of caspase-3 was still very low. While the HIF-1 $\alpha$ overexpressing xenograft tumors were knocked down of $\beta$-catenin, the caspase- 3 expression showed a remarkable increase upon ${ }^{131} \mathrm{I}$ treatment $(\mathrm{P}<0.001)$. Moreover, the content of survivin was reduced more markedly $(\mathrm{P}<0.05)$ and the level of $\mathrm{Ki}-67$ was even lower $(\mathrm{P}<0.05)$. Also, the role of $\beta$-catenin in affecting the efficacy of radioactive iodine treatment was confirmed by directly using $\beta$-catenin overexpressing FTC cells instead of the HIF-1 $\alpha$ overexpressing ones in this immunohistochemical analysis.

Taken together, these results suggested how the genuinely effective radioiodine treatment, which may guarantee normal functioning of NIS in iodide uptake (Fig. 1C), would intervene in the proliferative and apoptotic regulation of thyroid cancer cells.

Cell distribution of $\beta$-catenin and NIS are negatively correlated in xenograft tumors generated from FTC cells. To further verify in vivo that the impact of HIF-1 $\alpha$ overexpression on the efficiency of radioiodine treatment should rely on the localization regulation of NIS in a $\beta$-catenin-dependent manner, we analyzed the distribution of NIS or $\beta$-catenin in xenograft tumors generated from FTC cells with HIF- $1 \alpha / \beta$-catenin overexpression, as well as those having further knockdown of $\beta$-catenin.

Immunohistochemistry showed that the nuclear translocation of $\beta$-catenin was significantly enhanced in HIF- $1 \alpha / \beta$-catenin overexpressing tumor cells $(\mathrm{P}<0.05)$ (Fig. 5). Moreover, cytomembrane localization of NIS was markedly decreased $(\mathrm{P}<0.05)$. In addition, all these changes in the subcellular distribution of proteins were erased by shRNA-mediated knockdown of $\beta$-catenin. Thus, we infer more confidently that the restored localization of NIS, owing to reduced expression together with lessened nuclear localization of $\beta$-catenin, was indeed essential for the superior efficiency of ${ }^{131} \mathrm{I}$ treatment (Fig. 3A) as a provider of sufficient radioiodine uptake. 

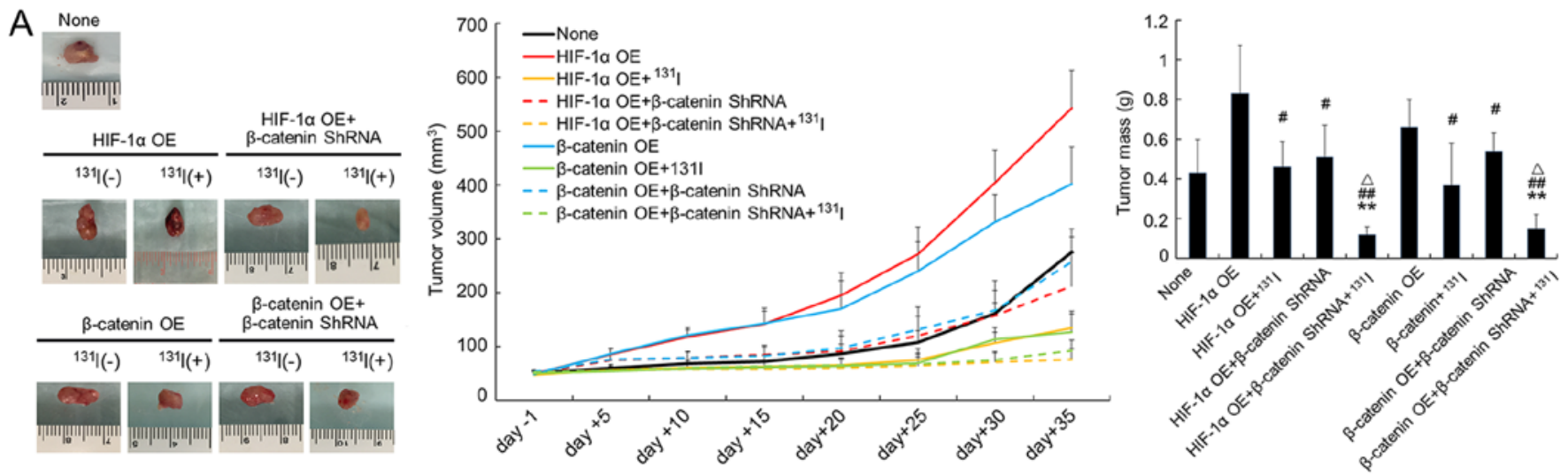

B

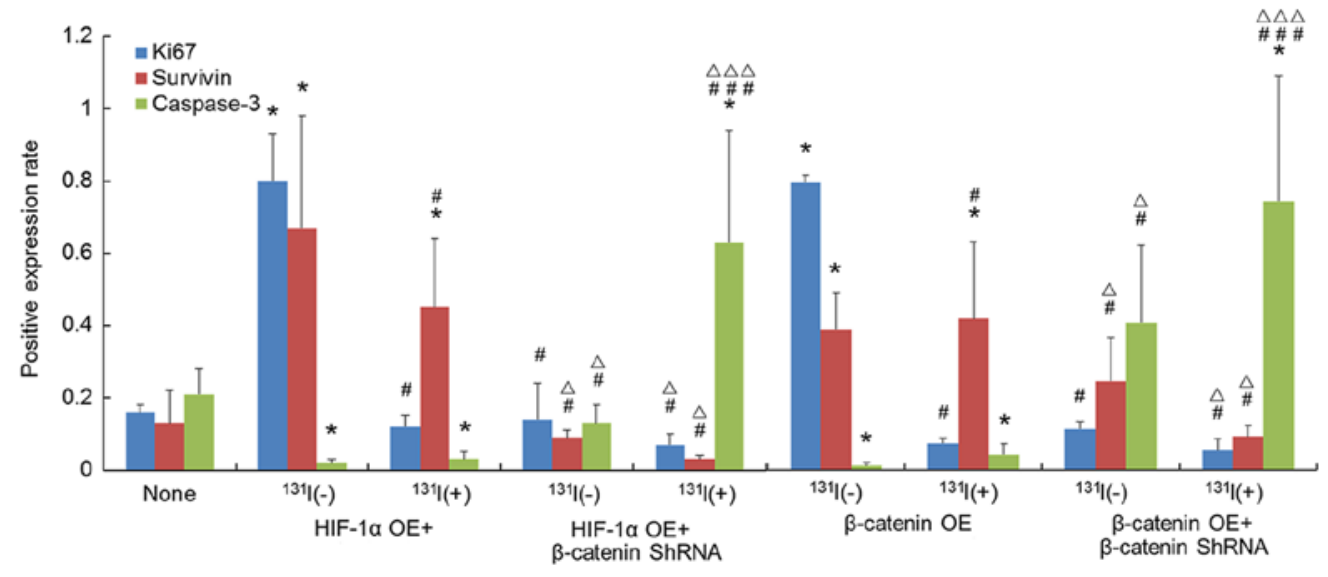

Figure 3. $\beta$-catenin knockdown can improve the growth inhibitory effect of radioiodine treatment in HIF-1 $\alpha$ overexpressing FTC cells. FTC133 cells with different treatments (grouping as Fig. 1) were injected into male SCID mice to measure the tumor growth. When xenograft tumor grew to the volume of $50 \mathrm{~mm}^{3}$, peritoneal injection of $37 \mathrm{MBq}{ }^{131} \mathrm{I}$ was introduced, while $0.1 \mathrm{ml}$ physical saline was used as control in all the groups that did not receive radioiodine treatment. There were altogether 9 groups as shown in the horizontal axis in right panel of (A). (A) From left to right are the representative images of tumors, the tumor growth curves, and the tumor mass 35 days after the ${ }^{131} \mathrm{I}$ treatment (or saline injection) in each group. The images were taken on the same day as tumor mass measurement. (B) Quantification (based on immunohistochemical staining, with representative images shown in Fig. 4) and statistics of the rate of Ki-67, survivin or caspase-3 expression in tumor tissues from different groups. For every index, 3 images of cancer tissues from each animal were selected for quantification. In addition, 5 high power fields were selected from each image for the analysis. $y$-axis indicates the average percentage of the positively stained cells from all fields of observation. For each assay, $\mathrm{n}=10$ (number of animals) in each group. ${ }^{*} \mathrm{P}<0.05$ and ${ }^{* *} \mathrm{P}<0.01$, vs. the None group. ${ }^{\#} \mathrm{P}<0.05$, ${ }^{\# \prime} \mathrm{P}<0.01$ and ${ }^{\# \# \#} \mathrm{P}<0.001$, vs. the same $\mathrm{OE}$ groups without $\beta$-catenin shRNA knockdown nor ${ }^{131} \mathrm{I}$ treatment. ${ }^{\Delta} \mathrm{P}<0.05$ and ${ }^{\Delta \Delta \Delta} \mathrm{P}<0.001$, vs. the same $\mathrm{OE}$ groups with ${ }^{131} \mathrm{I}$ treatment but without $\beta$-catenin shRNA knockdown.

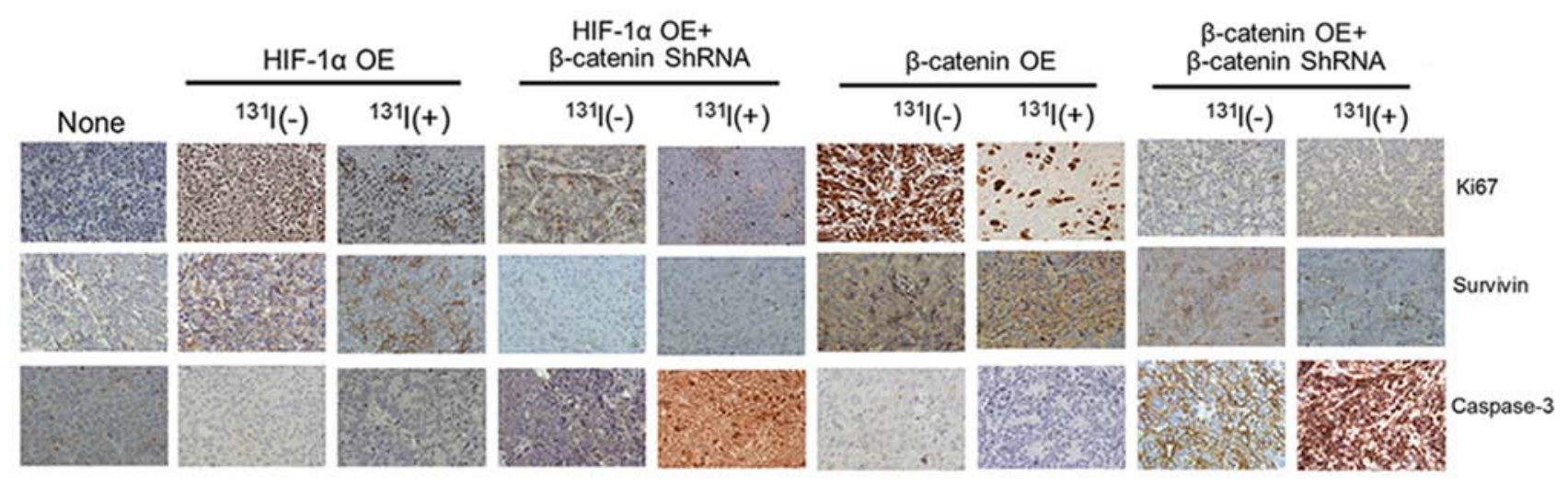

Figure 4. Representative immunohistochemical staining of Ki-67, survivin or caspase-3 expression in tumor tissues generated from FTC cells of different groups. Grouping was described in the legend of Fig. 3B. Magnification, x100.

\section{Discussion}

In the present study, we paralleled the interventions of HIF-1 $\alpha$ overexpression and $\beta$-catenin overexpression in thyroid carci- noma cell line FTC133, so as to demonstrate their impacts on the aggressiveness of cancer cells, the radioiodine uptake ability which is mediated by NIS, and the efficiency of radiotherapy towards xenograft tumors. By means of $\beta$-catenin 

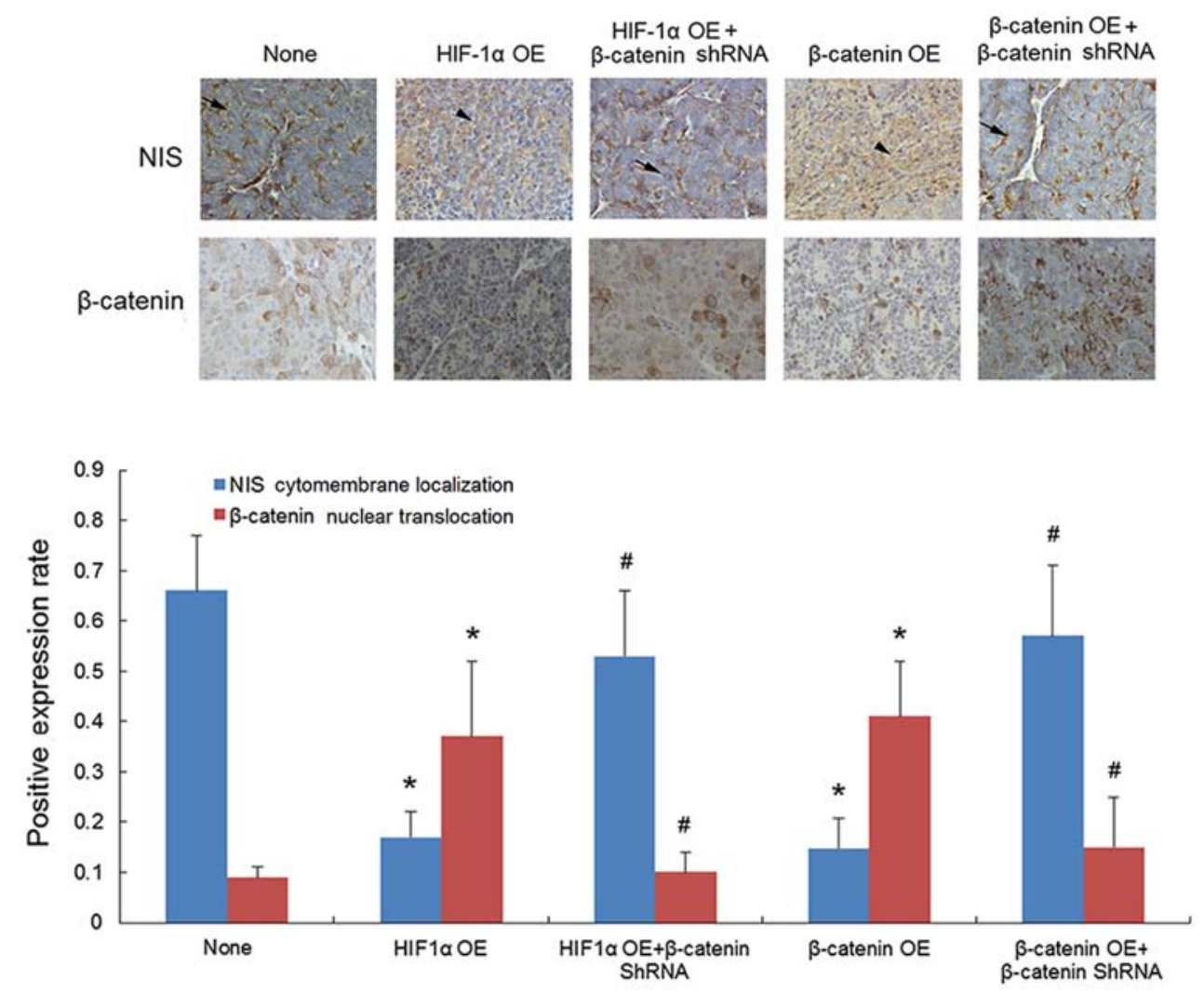

Figure 5. Cell distribution of $\beta$-catenin and NIS were negatively correlated in xenograft tumors generated from FTC cells. FTC133 cells with different treatments (grouping as Fig. 1) were injected into male SCID mice to measure the tumor growth. In 35 days after the xenograft tumor had grown to the volume of $50 \mathrm{~mm}^{3}$, the subcutaneous tumor tissues were obtained for immunohistochemical analyses. The samples were then counterstained with hematoxylin to indicate the nucleus. Representative immunohistochemical staining of NIS or $\beta$-catenin in tumor tissues from different groups are shown in the upper panel. NIS distributed along the cell outline was considered as locating on the cell membrane. Arrows indicate the typical membrane location while arrowheads indicate typical cytoplasmic location. Magnification, x100. For each index, 3 images of cancer tissues from each animal were selected for quantification. In addition, 5 high power fields were selected from each image for the analysis. Quantitative results and statistics are shown in the bottom and y-axis indicates the average percentage of the positively stained cells (membrane staining of the NIS or nuclear staining of the $\beta$-catenin) from all fields of observation. For each assay, $\mathrm{n}=10$ (number of animals) in each group. ${ }^{*} \mathrm{P}<0.05$ vs. the None group. ${ }^{~} \mathrm{P}<0.05$ vs. the same $\mathrm{OE}$ groups without $\beta$-catenin shRNA knockdown.

knockdown subsequent to HIF-1 $\alpha$ overexpression, we also confirmed the dependent relationship of these two factors in above-mentioned regulatory mechanisms.

In FTC133 cells which were originally derived from metastatic FTC (36) we hardly detected any expression of HIF-1 $\alpha$ (Fig. 1A). Yet, previous study had revealed that HIF-1 $\alpha$ was highly expressed even in primary thyroid cancers including FTC (34). We inferred that culture conditions in vitro may lead to impaired metastatic ability through inactivation of relevant signaling pathways. Once the expression of HIF-1 $\alpha$ was artificially upregulated, FTC cells cultured in vitro exhibited stronger growth ability together with more obvious metastatic propensity (Fig. 2). We deemed that overexpression of HIF-1 $\alpha$ may have made the FTC133 cells better mimicking tumor cells of aggressive thyroid carcinomas in vivo. In such FTC cells, we had observed that HIF-1 $\alpha$ acted upstream of $\beta$-catenin signaling (Fig. 1A), and confirmed by interference experiments that the aggressiveness of FTC cells endowed with HIF-1 $\alpha$ overexpression was $\beta$-catenin-dependent (Figs. 2 and 3). Those were supplementary proofs to previous discoveries on the relationship of the two pathways in tumor development $(23,25,29,30)$.

Previous observations pointed out that, FTC with aggressiveness may show resistance to radiotherapy. In addition, the fact that in MTC, inhibitors of HIF like 2ME2 is responsible for promoting the efficacy of anticancer drugs such as cabozantinib, had proved reversely the role of HIF-1 $\alpha$ in progression of tumors, increased aggressiveness and poor response to therapy (39). In the present study, we also found unsatisfactory efficiency of radioiodine treatment towards xenograft tumors generated from HIF-1 $\alpha / \beta$-catenin overexpressing FTC cells, which had exhibited metastatic propensity in vitro. While reducing the expression of $\beta$-catenin promoted the killing effect of ${ }^{131} \mathrm{I}$, reflecting the more rapid shrinkage of tumor volume together with tumor mass (Fig. 3A). We confirmed that the progression of xenograft tumors as a consequence of excessive proliferation and blocked apoptosis was thoroughly reduced when radiotherapy was introduced after $\beta$-catenin knockdown (Fig. 3B). Given that NIS subcellular localization and radioiodine uptake ability of FTC cells was profoundly subjected to HIF- $1 \alpha$ overexpression in a $\beta$-catenin-dependent manner (Fig. 1B and C), it is a reasonable explanation that in the xenograft thyroid tumors, the activated HIF1 $\alpha$ signaling and the impaired efficiency of radiotherapy was bridged by $\beta$-catenin induced NIS translocation. We corroborated this by revealing the relevance of $\beta$-catenin nuclear localization and NIS cytomembrane localization only in the xenograft tumor cells (Fig. 5). 
Previous studies have paid some attention to the expression level of NIS in cancer cells, which allows therapeutic application and prognosis estimation of radioiodine treatment, and our results suggested the location of NIS may likewise indicate the efficacy of radioiodine therapy. In fact, we even found that direct activation of $\beta$-catenin, as well as secondary to strengthened HIF- $1 \alpha$ signaling, would reduce the protein amount of NIS to a certain extent (Fig. 1A). While in a recent study, Sastre-Perona and Santisteban (24) found that in normal thyroid cells TSH and IGF-1 can facilitate $\beta$-catenin release from E-cadherin at the adherence junctions, and $\beta$-catenin will increase expression of NIS by elevating the transcriptional activity of Pax8. We supposed the discrepancy between our results and theirs may emerge on account of different downstream effectors, respectively, involved in physiological and pathological conditions.

Additionally, part from adequate protein content, the correct localization of NIS on thyroid cancer cells seems more pivotal in determining the capabilities and sensitivity of radiotherapy, for it has been reported that abundance of NIS was observed in differentiated thyroid cancer while the majority was in the cytoplasm with little on the cell surface membrane (40). Similar observations have also been obtained in breast cancers (13-15,19). Therefore, post-translational regulatory mechanisms, especially translocation of NIS, have been proposed as an important factor determining the functionality of NIS, and is of interest as a target to augment iodide uptake in NIS-expressing cancer cells. Riedel et al (41) explored the mechanism by which TSH regulates NIS distribution and found that NIS exhibits several consensus sites for the cAMPdependent protein kinase, protein kinase $\mathrm{C}$ and CK-2 kinases. They also revealed that the NIS phosphorylation pattern changes play a role in regulating its targeting from membrane to the intracellular compartments.

Yet overall, the exact regulatory mechanism of NIS especially on its localization is still not fully elucidated. To date, the present study is the first to connect the regulation of NIS both in subcellular localization and expression level, with the activated HIF-1 $\alpha$ signaling in tumor cells. We established a regulatory axis wherein $\beta$-catenin increased in response to HIF-1 $\alpha$ activation and plays an essential role in affecting NIS localization. Although in the context of thyroid tumors, the detailed regulatory mechanisms of NIS, downstream of $\beta$-catenin signaling remained unclear. We have evidence strongly suggesting the reduced efficiency of radioiodine treatment towards HIF-1 $\alpha$ overexpressing thyroid tumor cells may be heavily due to abnormal NIS localization caused by $\beta$-catenin activation. Thus, the radioiodine resistance of aggressive thyroid carcinomas which displayed highly activated HIF- $1 \alpha / \beta$-catenin signaling may be explained. Nevertheless, whether the translocation of NIS occurred parallelly or subsequently to the acquirement of aggressiveness (i.e. achieving EMT and possessing a high metastatic capacity), and indeed to what extent the efficiency of radioiodine therapy was determined by the NIS subcellular localization need to be answered in future studies.

In conclusion, this study made a preliminary exploration to elucidate the signals taking part in the regulation of NIS function as related to thyroid cancers. Dissection of such signaling pathways may lead to resolution of the treat- ment of radioiodine-refractory thyroid cancer. Our results will contribute to improving the postoperative prognosis of thyroid cancers such as FTC, and these advances might also have profound implications for the treatment of breast cancers and other non-thyroidal cancers currently being assessed for potential NIS-mediated radioiodine therapy (20).

\section{Acknowledgements}

The present study was supported by grants from the National Natural Science Foundation of China (nos. 81372858, 30700968, 30901725 and 30800416).

\section{References}

1. Giannoula E, Iakovou I and Chatzipavlidou V: Risk factors and the progression of thyroid malignancies. Hell J Nucl Med 18: 275-284, 2015 (In Greek).

2. Mohamed AF, González JM and Fairchild A: Patient benefit-risk tradeoffs for radioactive iodine-refractory differentiated thyroid cancer treatments. J Thyroid Res 2015: 438235, 2015.

3. Ferrari SM, Fallahi P, Politti U, Materazzi G, Baldini E, Ulisse S, Miccoli P and Antonelli A: Molecular targeted therapies of aggressive thyroid cancer. Front Endocrinol (Lausanne) 6: 176, 2015.

4. Luo Y, He DL, Ning L, Shen SL, Li L, Li X, Zhau HE and Chung LW: Over-expression of hypoxia-inducible factor-1alpha increases the invasive potency of LNCaP cells in vitro. BJU Int 98: 1315-1319, 2006

5. Steinert HC and Aberle S: CME: Radioactive iodine therapy in thyroid cancer. Praxis (Bern 1994) 104: 1235-1243; quiz 12441235, 2015 (In German).

6. Dai G, Levy O and Carrasco N: Cloning and characterization of the thyroid iodide transporter. Nature 379: 458-460, 1996.

7. Smanik PA, Liu Q, Furminger TL, Ryu K, Xing S, Mazzaferri EL and Jhiang SM: Cloning of the human sodium lodide symporter. Biochem Biophys Res Commun 226: 339-345, 1996.

8. Matsuno A, Murakami M, Hoya K, Yamada SM, Miyamoto S, Yamada S, Son JH, Nishido H, Ide F, Nagashima $\mathrm{H}$, et al: Clinicopathological and molecular histochemical review of skull base metastasis from differentiated thyroid carcinoma. Acta Histochem Cytochem 46: 129-136, 2013.

9. Bauer AJ: Thyroid nodules and differentiated thyroid cancer. Endocr Dev 26: 183-201, 2014.

10. Dadu R and Cabanillas ME: Optimizing therapy for radioactive iodine-refractory differentiated thyroid cancer: Current state of the art and future directions. Minerva Endocrinol 37: 335-356, 2012.

11. Rahmani N, Abbas Hashemi S, Fazli M and Raisian M: Clinical management and outcomes of papillary, follicular and medullary thyroid cancer surgery. Med Glas (Zenica) 10: 164-167, 2013.

12. Phay JE and Ringel MD: Metastatic mechanisms in follicular cell-derived thyroid cancer. Endocr Relat Cancer 20: R307-R319, 2013.

13. Dohán O, Baloch Z, Bánrévi Z, Livolsi V and Carrasco N: Rapid communication: Predominant intracellular overexpression of the $\mathrm{Na}^{+} / \mathrm{I}^{-}$symporter (NIS) in a large sampling of thyroid cancer cases. J Clin Endocrinol Metab 86: 2697-2700, 2001.

14. Saito T, Endo T, Kawaguchi A, Ikeda M, Katoh R, Kawaoi A, Muramatsu A and Onaya T: Increased expression of the sodium/ iodide symporter in papillary thyroid carcinomas. J Clin Invest 101: 1296-1300, 1998.

15. Wapnir IL, van de Rijn M, Nowels K, Amenta PS, Walton K, Montgomery K, Greco RS, Dohán O and Carrasco N: Immunohistochemical profile of the sodium/iodide symporter in thyroid, breast, and other carcinomas using high density tissue microarrays and conventional sections. J Clin Endocrinol Metab 88: $1880-1888,2003$.

16. Venkataraman GM, Yatin M, Marcinek R and Ain KB: Restoration of iodide uptake in dedifferentiated thyroid carcinoma: Relationship to human $\mathrm{Na}^{+} / \mathrm{I}$-symporter gene methylation status. J Clin Endocrinol Metab 84: 2449-2457, 1999.

17. Arturi F, Russo D, Schlumberger M, du Villard JA, Caillou B, Vigneri P, Wicker R, Chiefari E, Suarez HG and Filetti S: Iodide symporter gene expression in human thyroid tumors. J Clin Endocrinol Metab 83: 2493-2496, 1998. 
18. Dohán O, De la Vieja A, Paroder V, Riedel C, Artani M, Reed M, Ginter CS and Carrasco N: The sodium/iodide Symporter (NIS): Characterization, regulation, and medical significance. Endocr Rev 24: 48-77, 2003

19. Arturi F, Russo D, Giuffrida D, Schlumberger M and Filetti S: Sodium-iodide symporter (NIS) gene expression in lymph-node metastases of papillary thyroid carcinomas. Eur J Endocrinol 143: 623-627, 2000.

20. Boelaert K and Franklyn JA: Sodium iodide symporter: A novel strategy to target breast, prostate, and other cancers? Lancet 361 : 796-797, 2003.

21. Xing M: Molecular pathogenesis and mechanisms of thyroid cancer. Nat Rev Cancer 13: 184-199, 2013.

22. Zhang Z, Liu D, Murugan AK, Liu Z and Xing M: Histone deacetylation of NIS promoter underlies BRAF V600E-promoted NIS silencing in thyroid cancer. Endocr Relat Cancer 21: 161-173, 2014.

23. Guo H, Xing Y, Liu Y, Luo Y, Deng F, Yang T, Yang K and Li Y: Wnt/ $\beta$-catenin signaling pathway activates melanocyte stem cells in vitro and in vivo. J Dermatol Sci 83: 45-51, 2016.

24. Sastre-Perona A and Santisteban P: Wnt-independent role of $\beta$-catenin in thyroid cell proliferation and differentiation. Mol Endocrinol 28: 681-695, 2014

25. Lan L, Deng W, Chen HL, Huo LL, Deng LL, Zhang GY and Luo Y: All-trans retinoic acid improves iodine uptake of thyroid cancer cells via repressing transcriptional activity of $\beta$-catenin. Zhonghua Yi Xue Za Zhi 96: 553-558, 2016 (In Chinese).

26. Rossi ED, Straccia P, Palumbo M, Stigliano E, Revelli L, Lombardi CP, Santeusanio G, Pontecorvi A and Fadda G Diagnostic and prognostic role of HBME-1, galectin-3, and $\beta$-catenin in poorly differentiated and anaplastic thyroid carcinomas. Appl Immunohistochem Mol Morphol 21: 237-241, 2013.

27. Lan L, Luo Y, Cui D, Shi BY, Deng W, Huo LL, Chen HL, Zhang GY and Deng LL: Epithelial-mesenchymal transition induces cancer stem cell generation in human thyroid cancer cells in vitro. Zhonghua Yi Xue Za Zhi 93: 1261-1265, 2013 (In Chinese).

28. Pagni F, Manzoni M, Buscone S and Leone BE: $\beta$-catenin as a morpho-immunohistochemical marker for the diagnosis of papillary thyroid carcinoma. Arch Pathol Lab Med 139: 572-574, 2015.

29. Lan L, Deng W, Chen H, Huo L, Deng L, Zhang G and Luo Y: Nuclear translocation of $\beta$-catenin represses membrane localization of NIS in human thyroid cancer cells. Zhonghua Yi Xue Za Zhi 96: 891-896, 2016 (In Chinese).
30. Jin S, Borkhuu O, Bao W and Yang YT: Signaling pathways in thyroid cancer and their therapeutic implications. J Clin Med Res 8: 284-296, 2016.

31. Höckel M and Vaupel P: Tumor hypoxia: Definitions and current clinical, biologic, and molecular aspects. J Natl Cancer Inst 93: 266-276, 2001

32. Zhang Q, Bai X, Chen W, Ma T, Hu Q, Liang C, Xie S, Chen C, $\mathrm{Hu} \mathrm{L}, \mathrm{Xu} \mathrm{S}$, et al: Wnt/B-catenin signaling enhances hypoxiainduced epithelial-mesenchymal transition in hepatocellular carcinoma via crosstalk with hif-1 $\alpha$ signaling. Carcinogenesis 34: 962-973, 2013.

33. Burrows N, Babur M, Resch J, Williams KJ and Brabant G: Hypoxia-inducible factor in thyroid carcinoma. J Thyroid Res 2011: 762905, 2011.

34. Burrows N, Resch J, Cowen RL, von Wasielewski R, Hoang-Vu C, West CM, Williams KJ and Brabant G: Expression of hypoxiainducible factor 1 alpha in thyroid carcinomas. Endocr Relat Cancer 17: 61-72, 2010.

35. Masoud GN and Li W: HIF-1 $\alpha$ pathway: Role, regulation and intervention for cancer therapy. Acta Pharm Sin B 5: 378-389, 2015.

36. Goretzki PE, Frilling A, Simon D and Roeher HD: Growth regulation of normal thyroids and thyroid tumors in man. Recent Results Cancer Res 118: 48-63, 1990.

37. Zhao JH, Luo Y, Jiang YG, He DL and Wu CT: Knockdown of $\beta$-catenin through shRNA cause a reversal of EMT and metastatic phenotypes induced by HIF-1 $\alpha$. Cancer Invest 29: 377-382, 2011.

38. Kraiem Z, Sadeh O and Yosef M: Iodide uptake and organification, tri-iodothyronine secretion, cyclic AMP accumulation and cell proliferation in an optimized system of human thyroid follicles cultured in collagen gel suspended in serum-free medium. J Endocrinol 131: 499-506, 1991.

39. Lin H, Jiang X, Zhu H, Jiang W, Dong X, Qiao H, Sun X and Jiang H: 2ME2 inhibits the activated hypoxia-inducible pathways by cabozantinib and enhances its efficacy against medullary thyroid carcinoma. Tumour Biol 37: 381-391, 2016.

40. Zhang R, Wang H, Zhao J, Yao J, Shang H, Zhu H, Liao L and Dong J: Association between sodium iodide symporter and differentiated thyroid cancer: A meta-analysis of 9 studies. Int $\mathbf{J}$ Clin Exp Med 8: 17986-17994, 2015.

41. Riedel C, Levy O and Carrasco N: Post-transcriptional regulation of the sodium/iodide symporter by thyrotropin. J Biol Chem 276: 21458-21463, 2001 